\title{
P17: Vergleich zwischen subjektiver Beurteilung und objektiver Messung der Beleuchtungssituation in einer stationären Pflegeeinrichtung
}

\author{
Mirko Lezock
}

Online publiziert: 30. Oktober 2013

(C) Springer-Verlag Wien 2013

Einleitung/Hintergrund: Der Sturzrisikofaktor ,geringe Beleuchtung' ist bisher noch nicht durch Untersuchungen belegt. Dennoch hat die Beleuchtung Einfluss auf die Gesundheit und Sicherheit von Patienten und Mitarbeiter gleichermaßen.

Zielstellung/Methodik: Aufgrund der häufig unterschätzten, aber hohen Praxisrelevanz von Licht und Beleuchtung im Gesundheitswesen war das Ziel der Untersuchung, über eine schriftliche Befragung und Beleuchtungsmessung einen Vergleich zwischen subjektiver Beurteilung und objektiver Messung der Beleuchtung herauszuarbeiten.

Ergebnisse: Die Beleuchtung in der Einrichtung wurde von den Pflegenden als durchweg positiv eingeschätzt. Die Mes- sung der Beleuchtung und die anschließende Auswertung mittels Checkliste ergab insbesondere Mängel in den Bereichen Zustand und Bedienfreundlichkeit der Beleuchtungsanlage. Bei Auswertung aller Bewertungskriterien wurden insgesamt 122,5 von 184 möglichen Punkten erreicht.

Schlussfolgerung: Im Vergleich von subjektiver mit objektiver Beurteilung der Beleuchtung zeigten sich deutliche Differenzen. So wird die Qualität der Beleuchtung trotz festgestellter Mängel vom Pflegepersonal überschätzt, die Wirkung der Beleuchtung aber unterschätzt. Weitere Untersuchungen müssen noch zeigen, inwieweit die verwendete Methodik und die genutzten Instrumente für diesen Vergleich geeignet sind.

M. Lezock $(\bowtie)$

Fakultät Gesundheits- und Pflegewissenschaften,

Westsächsische Hochschule Zwickau, Zwickau, Deutschland

E-Mail: Mirko.Lezock.0rr@fh-zwickau.de 